\title{
Pressão expiratória positiva nas vias aéreas não reproduz as respostas de frequência cardíaca à manobra de Valsalva em homens jovens saudáveis
}

\author{
Expiratory positive airway pressure does not reproduce heart \\ rate responses to Valsalva maneuver in healthy young men \\ Isabella Gracindo Pissinato', Marlus Karsten², Laura Maria Tomazi Neves³, \\ Vinicius Minatel ${ }^{4}$, Audrey Borghi-Silva ${ }^{5}$, Aparecida Maria Catai ${ }^{5}$
}

RESUMO I A pressão expiratória positiva nas vias aéreas (EPAP) é um recurso terapêutico que compreende uma inspiração seguida de expiração contra resistência. Sua aplicação promove ajustes no sistema cardiovascular, de maneira similar ao observado durante a manobra de Valsalva (MV). O objetivo deste estudo foi analisar a resposta da frequência cardíaca (FC) à MV e às diferentes formas de aplicação de EPAP a fim de identificar se e em qual condição esta técnica reproduz a resposta da FC observada na MV, em homens jovens aparentemente saudáveis. Foram estudados 10 sujeitos ( $24 \pm 3$ anos; $25 \pm 3 \mathrm{~kg} / \mathrm{m}^{2}$ ) que realizaram os procedimentos de MV e EPAP, aleatoriamente em dias diferentes. Na MV o esforço expiratório foi sustentado por $15 \mathrm{~s}$ (pressão oral de $4 \mathrm{O}$ mmHg [53,4 cmH $\mathrm{CH}_{2} \mathrm{O}$ ). Empregouse duas técnicas de EPAP (isolada e terapêutica) contra 3 níveis de pressão $\left(10,15\right.$ e $\left.2 \mathrm{O} \mathrm{cmH}_{2} \mathrm{O}\right)$, aplicados aleatoriamente. As manobras foram repetidas três vezes com intervalo de cinco minutos. Considerou-se o maior valor de variação da FC (DFC) de cada manobra para análise. Empregou-se o teste Shapiro-Wilk para verificar a distribuição dos dados e ANOVA para medidas repetidas, com post-hoc de Fisher, considerando-se $\alpha<0,05$. Os valores de DFC observados na MV foram maiores $(p<0,05)$ que os encontrados nas diferentes técnicas de EPAP, independentemente do nível

\begin{abstract}
I The expiratory positive airway pressure (EPAP) is a therapeutic resource that comprises an inspiration followed by expiration against resistance. During its application there were adjustments in the cardiovascular system, similar to those observed during the Valsalva maneuver (VM). The aim of this study was to analyze the heart rate (HR) response to VM and to different ways of EPAP application to identify if and in which condition this technique reproduces the HR response observed in the VM, in apparently healthy young men. Ten subjects $\left(24 \pm 3\right.$ years, $\left.25 \pm 3 \mathrm{~kg} / \mathrm{m}^{2}\right)$ performed randomly the $\mathrm{VM}$ and EPAP procedures on different days. The expiratory effort in VM was sustained for $15 \mathrm{~s}$ (oral pressure of $40 \mathrm{mmHg}$ $\left[53.4 \mathrm{~cm} \mathrm{H}_{2} \mathrm{O}\right]$ ). Two EPAP techniques were employed (alone and therapeutic) against three pressure levels (10, 15 and $2 \mathrm{O} \mathrm{cmH}_{2} \mathrm{O}$ ), randomly applied. The maneuvers were repeated three times with five minutes interval. It was considered the greatest value of HR variation (DHR) for each maneuver analysis. Were used the Shapiro-Wilk test to analyze the data distribution and the ANOVA for repeated measures, with Fisher's post-hoc, considering $\alpha<0.05$. The DHR values observed in VM were higher $(p<0.05)$ than those found in the different EPAP techniques, regardless the pressure level employee. The EPAP application, in
\end{abstract}

\footnotetext{
Estudo desenvolvido no Laboratório de Fisioterapia Cardiovascular, Núcleo de Pesquisa em Exercício Físico (NUPEF), Departamento de Fisioterapia, Universidade Federal de São Carlos (UFSCar) - São Carlos (SP), Brasil.

'Fisioterapeuta; Residente do Programa de residência em Fisioterapia Hospitalar do Hospital e Maternidade Celso Pierro da Pontifícia Universidade Católica de Campinas PUC-Campinas - Campinas (SP), Brasil.

${ }^{2}$ Fisioterapeuta; Doutor em Fisioterapia pelo Programa de Pós-graduação em Fisioterapia da UFSCar - São Carlos (SP), Brasil. ${ }^{3}$ Fisioterapeuta ; Mestre; Doutoranda pelo programa de Ciências e Tecnologias em Saúde da Universidade de Brasília UnB - Brasília (DF), Brasil.

${ }^{4}$ Fisioterapeuta; Mestrando do Programa de Pós-graduação em Fisioterapia da Universidade Federal de São Carlos (UFSCar) São Carlos (SP), Brasil.

${ }^{5}$ Fisioterapeuta; Doutora; Docente do Programa de pós-graduação em Fisioterapia da UFSCar - São Carlos (SP), Brasil.
} 
pressórico empregado. A aplicação de EPAP, nos três níveis pressóricos, gera menor sobrecarga cardíaca e não reproduz as respostas da FC observadas na MV.

Descritores | respiração com pressão positiva; músculos respiratórios; sistema nervoso autônomo; modalidades de Fisioterapia. these three level pressures, generates less cardiac overload and does not reproduce HR responses observed in the VM.

Keywords I positive-pressure respiration; respiratory muscles; autonomic nervous system; Physical Therapy modalities.

\section{INTRODUÇÃO}

A pressão expiratória positiva nas vias aéreas (EPAP) é considerada a técnica mecânica mais simples empregada para reexpansão pulmonar, em virtude da facilidade para geração e oferta de pressão positiva expiratória (PEEP) em respiração espontânea. A EPAP tem diferentes indicações, sendo amplamente empregada na prevenção e/ou tratamento de disfunções respiratórias associadas a condições cirúrgicas ou traumáticas (atelectasia, pneumonia, derrame pleural e pneumotórax), no tratamento de limitação crônica ao fluxo aéreo (doença pulmonar obstrutiva crônica e asma) e no treinamento de força e resistência dos músculos expiratórios ${ }^{1-3}$.

A execução da EPAP é caracterizada por inspiração seguida de expiração contra resistência pressórica linear. A profundidade da inspiração relaciona-se com o nível do esforço expiratório, tendo em vista a necessidade de maior inflação pulmonar. Durante o esforço expiratório pode ocorrer elevação abrupta e voluntária das pressões intratorácica e intra-abdominal, de maneira similar ao observado durante a manobra de Valsalva (MV).

Os efeitos mecânicos gerados na caixa torácica e no sistema cardiovascular durante a MV e EPAP são mediados pelos receptores de estiramento pulmonar, mecanoceptores cardiopulmonares, barorreceptores arteriais e quimioceptores ${ }^{4,5}$. Então, as respostas são integradas no sistema nervoso central e desencadeiam a ativação dos sistemas simpático e parassimpático, que modulam o sistema cardiovascular, em especial a frequência cardíaca (FC) e a pressão arterial (PA) ${ }^{4,5}$.

O conjunto de respostas que ocorre durante e após a execução da MV está amplamente descrito na literatura e é dividido em quatro fases ${ }^{6}$ : Fase I - no início da manobra observa-se um aumento da pressão intratorácica, elevação transitória da PA e queda da FC; Fase II - a manutenção da pressão intratorácica elevada dificulta o retorno venoso, resultando em uma queda da PA e, reflexamente, observa-se uma elevação da FC e da resistência vascular periférica (RVP) para restaurar os níveis basais da PA; Fase III - ao final da MV ocorre, simultaneamente, a queda da pressão intratorácica e da $\mathrm{PA}$, aumento do retorno venoso e taquicardia devido à ação dos barorreceptores e mecanoceptores dos vasos pulmonares; Fase IV - elevação súbita da PA a valores superiores aos de repouso. Esse aumento da PA estimula os barorreceptores que consequentemente aumentam o tônus vagal e inibem os eferentes simpáticos, provocando bradicardia reflexa e vasodilatação arteriolar que resultará em diminuição da $\mathrm{FC}$ a valores inferiores aos observados em repouso ${ }^{4,5,7}$.

Embora sejam relatadas poucas contraindicações ao emprego de EPAP na prática clínica, em virtude da realização de um esforço expiratório contra resistência, pode haver relação entre a aplicação da EPAP e a realização da MV em termos de sobrecarga cardíaca, uma vez que durante a MV ocorre diminuição do retorno venoso, da circulação pulmonar e do fluxo sanguíneo coronariano devido ao aumento da pressão intratorá$\mathrm{cica}^{5,7,8}$. A hipótese do estudo é que a EPAP reproduz as respostas observadas da frequência cardíaca na $\mathrm{MV}$ em jovens saudáveis, independentemente do modo e da pressão empregada. Assim, o objetivo principal do estudo foi analisar as respostas da FC à aplicação da EPAP, a fim de identificar se e em qual condição a aplicação de EPAP reproduz o comportamento observado na MV, em jovens saudáveis.

\section{MÉTODOS}

\section{Sujeitos}

O estudo seguiu as diretrizes da Resolução CNS no 196/96 e foi aprovado pelo Comitê de Ética em Pesquisa da Universidade Federal de São Carlos (UFSCar) (Parecer no 064/2010). Todos os voluntários que aceitaram participar do estudo assinaram o termo de consentimento livre e esclarecido. Os 
procedimentos experimentais foram realizados no Laboratório de Fisioterapia Cardiovascular do Núcleo de Pesquisa em Exercício Físico (NUPEF), do Departamento de Fisioterapia da UFSCar.

Estudantes universitários do sexo masculino com idade entre 21 e 29 anos foram convidados a participar do estudo. Os critérios de inclusão empregados foram: índice de massa corpórea (IMC) entre 18,5 e $29,9 \mathrm{~kg} / \mathrm{m}^{2}$, ausência de doenças diagnosticadas, tabagismo, terapia medicamentosa ou alterações eletrocardiográficas em repouso. Foram empregados os seguintes critérios de exclusão: presença de alterações clínicas ou eletrocardiográficas durante ou após a realização dos procedimentos experimentais, dificuldade técnica de realização dos procedimentos experimentais e dificuldade de adaptação à máscara facial.

\section{Procedimentos experimentais}

Os voluntários foram orientados a não ingerir bebidas alcoólicas e/ou estimulantes por pelo menos 12 horas antes do teste, fazer refeição leve até duas horas antes dos procedimentos, evitar exercícios extenuantes nas 24 horas prévias ao teste e ter um bom período de sono na noite anterior ao dia da coleta de dados. Dois dias antes da coleta dos dados foram executados procedimentos de familiarização do voluntário com os equipamentos e procedimentos. Todos os procedimentos foram executados em um mesmo período do dia, em sala com temperatura e umidade relativa do ar controladas, respectivamente, entre 22 e $24^{\circ} \mathrm{C}$, e 40 e $60 \%$.

Os eletrodos foram posicionados na derivação MC5 e os voluntários foram acomodados na posição supina por um período de 10 minutos para identificação da FC e PA em repouso. A captação e o processamento dos sinais eletrocardiográficos foram feitos por meio de um monitor cardíaco de um canal (TC-500, ECAFIX, São Paulo, SP, Brasil) acoplado a um conversor analógico digital Lab-PC+ (National Instruments Co, Austin, TX, USA). Assim, o sinal analógico do eletrocardiograma pode ser acessado por um programa de processamento dos sinais digitalizados 9 . As medidas de PA foram realizadas antes do início da manobra (repouso) e imediatamente após o final de cada manobra, pelo método auscultatório, utilizando-se esfigmomanômetro de coluna de mercúrio (Sankey, Brasil) e estetoscópio (3M, Littmann Cardiology, EUA).

Os sujeitos realizaram os procedimentos de MV e EPAP na postura sentada, em dias diferentes e em ordem aleatorizada. O esquema de execução dos procedimentos experimentais está representado na Figura 1.

\section{Manobra de Valsalva}

A MV foi realizada após um período de 60 segundos em repouso, no qual os voluntários deveriam manter frequência respiratória (FR) entre 12 e 15 ciclos por minuto. Para a realização da manobra foi empregada uma peça bucal conectada por um tubo semirrígido de $400 \mathrm{~mm}$ de comprimento e cinco $\mathrm{mm}$ de diâmetro a um manômetro (DIASYST SM 1000, SWISSBRAS, SP, BRASIL) (Figura 2A). O escape de ar foi evitado pelo uso de clipe nasal e pelo acoplamento adequado entre a peça bucal e a boca. Para garantir a abertura da glote durante a execução da manobra, foi utilizada uma peça bucal com orifício de 2 $\mathrm{mm}$ para escape de $a r^{5}$.

Após receberem um sinal sonoro, os sujeitos realizavam uma inspiração lenta e profunda seguida de esforço expiratório controlado ( $40 \mathrm{mmHg}$ ) com duração de $15 \mathrm{~s}$ (Figura 3I). Esta manobra foi repetida três vezes com intervalo de cinco minutos entre elas. Para avaliação da correta execução da MV foram observados, durante a

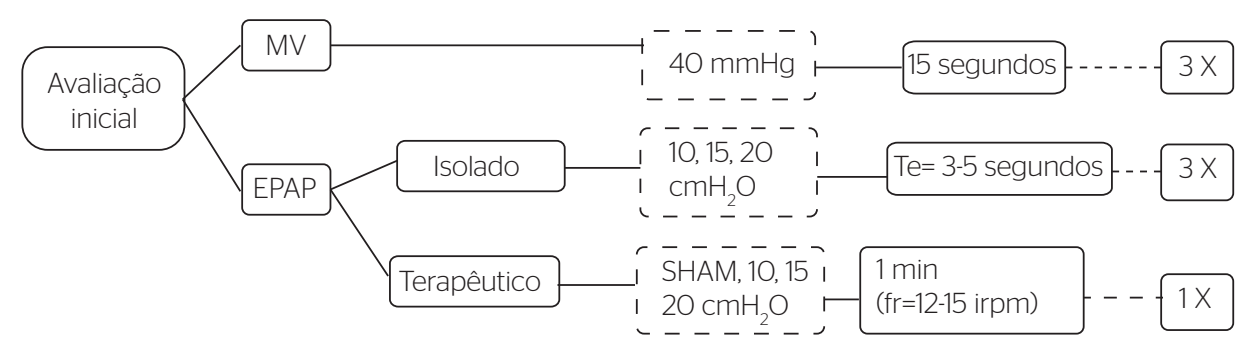

MV: manobra de Valsalva; EPAP: pressão expiratória positiva nas vias aéreas; SHAM: ausência de válvula resistora; fR: frequência respiratória; Te: tempo expiratório; irpm: incursões respiratórias por minuto; x: número de repetições 


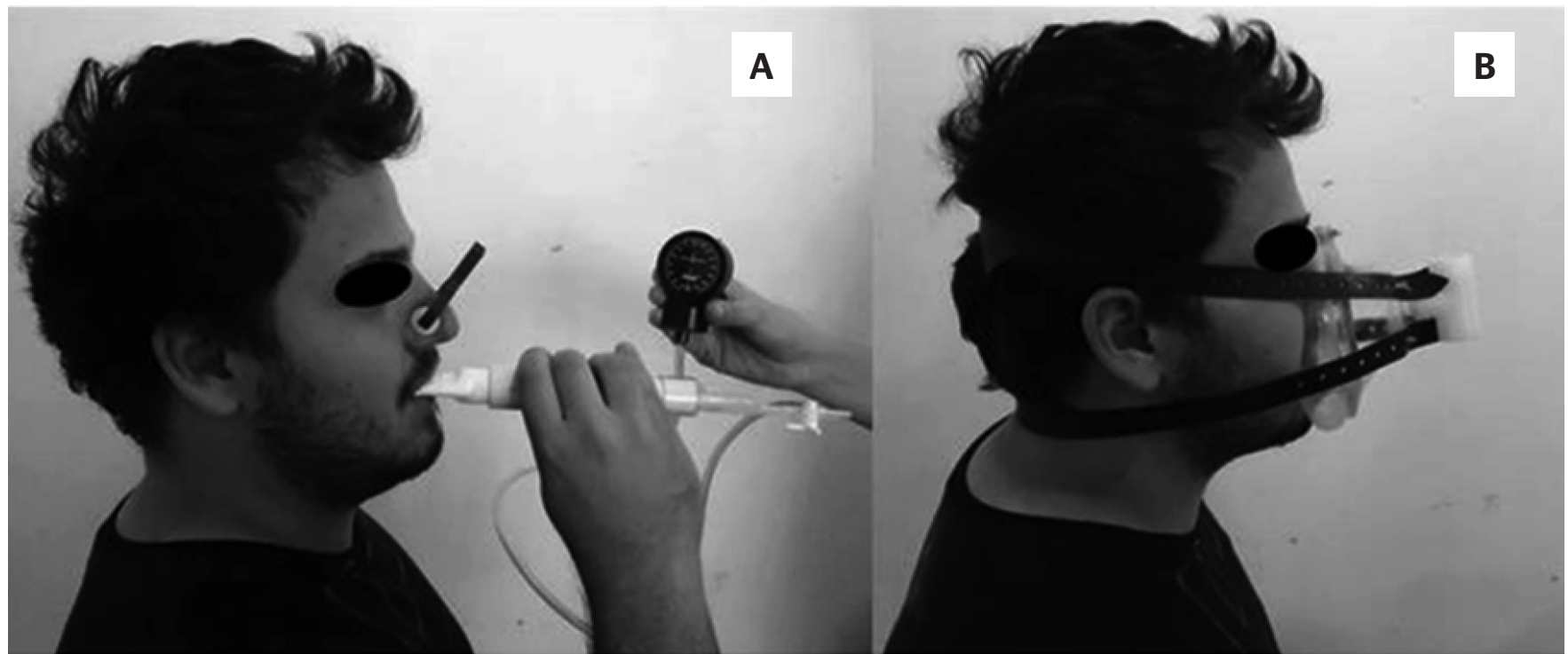

Figura 2. Voluntário durante a realização dos procedimentos: (A) manobra de Valsalva; (B) pressão expiratória positiva nas vias aéreas

execução da manobra, a manutenção da pressão no manômetro, a presença de rubor facial, estase jugular no pescoço ${ }^{4,10}$ e os movimentos da caixa torácica ${ }^{10}$ e, após a interrupção da manobra, a elevação rápida da FC seguida de bradicardia ${ }^{4}$.

\section{Pressão expiratória positiva nas vias aéreas}

A fim de isolar os possíveis efeitos intervenientes do uso da máscara, bem como promover a familiarização do voluntário com sua utilização, realizou-se uma única medida sem o uso da válvula resistora (SHAM), na qual o indivíduo, com a máscara acoplada ao seu rosto por um fixador cefálico, deveria manter sua respiração basal por cinco minutos. Foram aplicados dois modos de execução da EPAP (isolado e terapêutico), contra três níveis de pressão: 10, 15 e $20 \mathrm{cmH}_{2} \mathrm{O}$ (Figuras $2 \mathrm{~B}$, 3II e III). Entre cada uma das aplicações da técnica de EPAP, houve intervalo de cinco minutos.

$\mathrm{Na}$ forma isolada, após realização de inspiração lenta e profunda, os voluntários realizavam três manobras expiratórias contra cada um dos níveis de pressão, definidos em ordem aleatória, com tempo expiratório variando de três a cinco segundos. $\mathrm{Na}$ forma terapêutica, os voluntários permaneciam com a máscara acoplada ao rosto durante 1 minuto e realizavam de 10 a 15 ciclos respiratórios, contra cada um dos níveis de pressão, definidos em ordem aleatória. Este procedimento foi repetido uma única vez em cada um dos diferentes níveis pressóricos. O examinador, durante e após a execução das manobras, ficava atento acerca de sinais e sintomas, como palidez, sudorese, desconforto, vertigem, náuseas

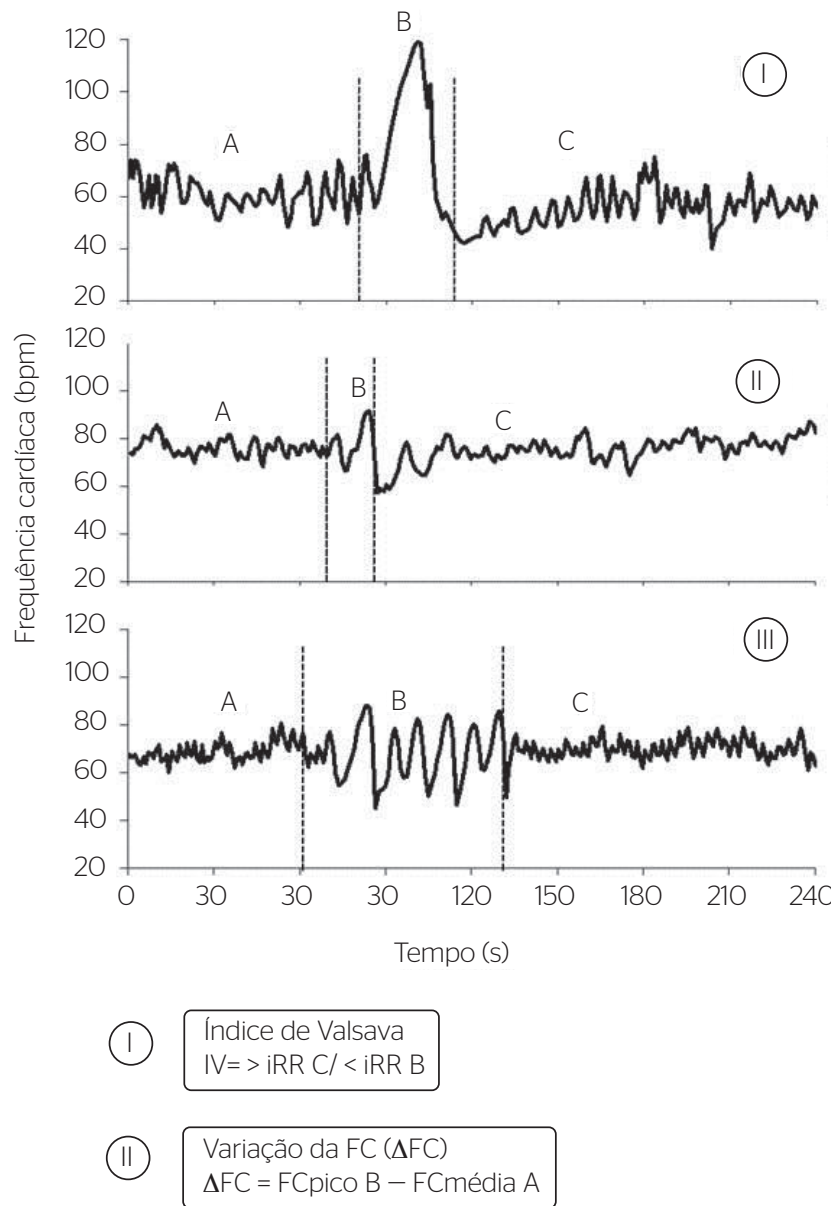

A: repouso; B: procedimentos; C: recuperação

Figura 3. Representação da resposta da FC durante a execução dos procedimentos: (I) manobra de Valsalva; (II) pressão expiratória positiva nas vias aéreas isolado, (III) pressão expiratória positiva nas vias aéreas terapêutico. 
e comportamento inesperado da pressão arterial sistêmica. Estas condições seriam indicativas de interrupção da execução da manobra.

\section{Análise dos dados}

Para a análise dos dados da FC, foram calculados o índice de Valsalva (IV, divisão do maior iR-R no período de recuperação pelo menor iR-R no período de taquicardia durante a $\mathrm{MV}$ ) e a variação da FC $(\Delta \mathrm{FC}$, diferença entre a maior $\mathrm{FC}$ durante os procedimentos e a FC média do período de repouso pré-manobra, correspondente a 60 segundos). A Figura 3 representa as respostas da FC durante a execução dos procedimentos e sua interpretação para análise dos dados.

\section{Análise estatística}

Os dados foram analisados com emprego do software Statistica7 (StatSoft Inc., Oklahoma, EUA). Com base nos resultados do estudo piloto $(n=5)$, estimamos que uma amostra de 10 indivíduos teria um poder estatístico de $80 \%$ para detectar uma diferença de 20 bpm

Tabela 1. Idade, características antropométricas, frequência cardíaca e pressão arterial em repouso e índice de Valsalva

\begin{tabular}{lc} 
Variáveis & Média $\pm \mathrm{DP}$ \\
\hline Idade (anos) & $23,9 \pm 3,0$ \\
Massa corporal (kg) & $79,1 \pm 10,2$ \\
Estatura (m) & $1,79 \pm 0,06$ \\
Índice de massa corporal $\left(\mathrm{kg} / \mathrm{m}^{2}\right)$ & $24,8 \pm 2,6$ \\
Frequência cardíaca em repouso (bpm) & $72 \pm 9$ \\
Pressão arterial sistólica em repouso (mmHg) & $120 \pm 1,75$ \\
Pressão arterial diastólica em repouso $(\mathrm{mmHg})$ & $83 \pm 1,89$ \\
Índice de Valsalva & $2,1 \pm 0,4$
\end{tabular}

na variação da frequência cardíaca $(\Delta \mathrm{FC})$. $\mathrm{O}$ nível de significância foi fixado em 5\%. Dados com distribuição normal (Shapiro-Wilk) foram apresentados como média \pm desvio padrão. A análise de variância de um fator (ANOVA one-way) com post-hoc de Fisher foi empregada para comparar a resposta da FC entre a MV e a EPAP, nas suas diferentes modalidades e pressões de aplicação, e para comparação da resposta da FC entre as diferentes pressões de aplicação $\left(10,15\right.$ e $20 \mathrm{cmH}_{2} \mathrm{O}$; e SHAM, na modalidade terapêutica) em cada modalidade da EPAP.

\section{RESULTADOS}

A caracterização da amostra (idade, características antropométricas, FC e PA de repouso, e índice de Valsalva [IV]) é apresentada na Tabela 1.

A Tabela 2 apresenta os maiores valores da variação de frequência cardíaca $(\triangle \mathrm{FC})$ e pressão arterial sistólica e diastólica ( $\triangle \mathrm{PAS}$ e $\triangle \mathrm{PAD})$ na manobra de Valsalva e na aplicação do EPAP nas modalidades isolada (10, 15 e $20 \mathrm{cmH}_{2} \mathrm{O}$ ) e terapêutica (SHAM, 10, 15 e 20 $\mathrm{cmH}_{2} \mathrm{O}$ ). $\mathrm{Na}$ análise dos dados obtidos durante a execução da EPAP foram encontrados valores que variaram de 8 a 28 bpm no modo isolado e de 4 a 27 bpm no modo terapêutico, incluindo o SHAM. Por sua vez, na $\mathrm{MV}$ os valores de $\triangle \mathrm{FC}$ oscilaram entre 20 e $67 \mathrm{bpm}$. A Figura 3 apresenta a $\triangle \mathrm{FC}$ durante a execução da técnica de EPAP e da MV em um voluntário representativo da amostra.

Em relação às respostas de $\mathrm{PA}$, não foram observadas diferenças estatísticas significativas entre as manobras. Houve, contudo, pequena variação das pressões arteriais sistólica e diastólica entre o período de repouso e o pico da manobra.

Tabela 2. Variação de frequência cardíaca e de pressão arterial sistólica e diastólica entre o repouso e o pico da manobra de Valsalva e da aplicação do pressão expiratória positiva nas vias aéreas nas modalidades isolada $\left(10,15\right.$ e $20 \mathrm{cmH}_{2} \mathrm{O}$ ) e terapêutica ( $\mathrm{SHAM}, 10,15$ e $20 \mathrm{cmH}_{2} \mathrm{O}$ )

\begin{tabular}{|c|c|c|c|c|c|c|c|}
\hline \multirow{2}{*}{\multicolumn{2}{|c|}{ Manobras }} & \multicolumn{2}{|c|}{ Variação da FC (bpm) } & \multicolumn{4}{|c|}{ Variação da PA (mmHg) } \\
\hline & & \multirow{2}{*}{$\begin{array}{c}\text { Modo isolado } \\
\qquad \mathrm{FC}\end{array}$} & \multirow{2}{*}{$\begin{array}{c}\begin{array}{c}\text { Modo } \\
\text { terapêutico }\end{array} \\
\Delta \mathrm{FC}\end{array}$} & \multicolumn{2}{|c|}{ Modo isolado } & \multicolumn{2}{|c|}{ Modo terapêutico } \\
\hline & & & & $\triangle \mathrm{PAS}$ & $\triangle \mathrm{PAD}$ & $\triangle \mathrm{PAS}$ & $\triangle \mathrm{PAD}$ \\
\hline MV & $53,4 \mathrm{cmH}_{2} \mathrm{O}$ & $41 \pm 10$ & $41 \pm 10$ & $8 \pm 4$ & $-1 \pm 7$ & $8 \pm 4$ & $-1 \pm 7$ \\
\hline \multirow{4}{*}{ EPAP } & SHAM & - & $12 \pm 6^{a, b}$ & - & - & $3 \pm 5$ & $\mathrm{O} \pm 3$ \\
\hline & $10 \mathrm{cmH}_{2} \mathrm{O}$ & $19 \pm 5$ & $15 \pm 6$ & $5 \pm 6$ & $2 \pm 2$ & $3 \pm 2$ & $2 \pm 7$ \\
\hline & $15 \mathrm{cmH}_{2} \mathrm{O}$ & $22 \pm 5$ & $17 \pm 6$ & $5 \pm 3$ & $2 \pm 2$ & $1 \pm 8$ & $\mathrm{O} \pm 5$ \\
\hline & $2 \mathrm{O} \mathrm{CmH}_{2} \mathrm{O}$ & $20 \pm 5$ & $19 \pm 7$ & $6 \pm 5$ & $2 \pm 3$ & $4 \pm 7$ & $2 \pm 2$ \\
\hline \multicolumn{2}{|c|}{ Valor $\mathrm{p}$} & $<0,001$ & $<0,001$ & 0,146 & 0,265 & 0,051 & 0,363 \\
\hline
\end{tabular}

$\triangle$ FC: frequência cardíaca; $\triangle \mathrm{PAS}$ : pressão arterial sistólica; $\triangle \mathrm{PAD}$ : pressão arterial diastólica; EPAP: pressão expiratória positiva nas vias aéreas; MV: manobra de Valsalva.

ANOVA one-way com post-hoc de Fisher foi empregada para comparar a resposta da FC e da PA na MV com as observadas nas diferentes pressões de aplicação de EPAP, em cada uma das modalidades, e para comparar a resposta da FC entre cada modalidadede aplicação de EPAP, onde ${ }^{a} \mathrm{p}=0,023\left(\mathrm{SHAM} \times 15 \mathrm{cmH}_{2} \mathrm{O}\right)$ e ${ }^{\mathrm{b}} \mathrm{p}=\mathrm{O}, 02 \mathrm{O}\left(\mathrm{SHAM} \times 2 \mathrm{O} \mathrm{cmH}_{2} \mathrm{O}\right)$. 


\section{DISCUSSÃO}

Os principais achados deste estudo indicam que as repercussões da FC durante a MV ocorrem em maior proporção comparativamente à EPAP, independente do nível de pressão utilizado e do modo de aplicação, isolado e terapêutico. Em relação à $\Delta \mathrm{FC}$, não houve diferença entre os diferentes níveis de pressões de EPAP no modo isolado. Contudo, na modalidade terapêutica do EPAP, a $\triangle \mathrm{FC}$ foi menor no nível SHAM em relação aos níveis de pressão 15 e $20 \mathrm{cmH}_{2} \mathrm{O}$.

$\mathrm{O}$ índice de Valsalva tem sido utilizado como uma medida da reatividade cardiovascular ${ }^{5,11,12}$. Esta, por sua vez, é definida como o padrão individual de respostas hemodinâmicas a agentes estressores e desempenha importante papel no desenvolvimento de doenças cardiovasculares ${ }^{13}$. No presente estudo, os valores do IV, que variaram entre 1,4 e 2,8, mostram que os voluntários do presente estudo apresentam reatividade cardiovascular normal. Tais valores foram semelhantes aos valores encontrados em estudos realizados por outros grupos de pesquisa ${ }^{11,12}$ e em estudo prévio realizado em nosso laboratório, o qual mostrou que o IV apresenta menor valor entre os homens de meia-idade, quando comparados a jovens saudáveis ${ }^{4}$.

Neste estudo, observamos que a $\triangle \mathrm{FC}$ na $M V$ foi maior $(p<0,001)$ em relação à aplicação de EPAP (isolado e terapêutico). A $\triangle \mathrm{FC}$ é influenciada pelos ajustes hemodinâmicos que acontecem mediante o esforço expiratório contra resistência aplicada nas manobras estudadas ${ }^{10}$. Durante a MV, esses ajustes parecem ocorrer com maior magnitude do que na aplicação de EPAP, o que, provavelmente, deve-se aos maiores valores pressóricos e duração da manobra, pois o tempo de execução da MV foi de três a cinco vezes maior do que na aplicação de EPAP.

Nesse sentido, um estudo recente ${ }^{14}$ avaliou as respostas cardiovasculares de tocadores de tuba que deveriam: i) tocar notas baixas, médias e altas por 15 segundos, e ii) executar a MV nas pressões de 10, 40 e $60 \mathrm{mmHg}$ pelo mesmo tempo. Durante a execução de notas altas os tocadores de tuba apresentaram respostas similares às encontradas na MV (40 e $60 \mathrm{mmHg}$ ). Entretanto, quando tocavam notas baixas as respostas cardiovasculares foram discretas, aproximando-se das respostas observadas na MV de $10 \mathrm{mmHg}$. A diferença existente entre a execução de notas altas e baixas é o padrão de fluxo expiratório adotado, sendo maior durante as notas baixas. O padrão de alto fluxo expiratório necessário para tocar as notas baixas é similar ao encontrado no esforço expiratório realizado durante as medidas de EPAP.
Os efeitos hemodinâmicos gerados pela aplicação de EPAP, independentemente do nível pressórico, podem ser explicados pelo aumento da pressão intratorácica, compressão das veias cava superior e inferior, redução do enchimento atrial e ventricular, e consequente diminuição do débito cardíaco ${ }^{15,16}$. No presente estudo não foi observada diferença estatística para a resposta da $\mathrm{FC}$ entre os diferentes níveis de pressão de EPAP, considerando-se os modos de aplicação isolado e terapêutico. Em ambos os modos de aplicação de EPAP os menores valores de $\Delta \mathrm{FC}$ foram encontrados durante a aplicação da pressão equivalente a $10 \mathrm{cmH}_{2} \mathrm{O}$. Resultado semelhante foi encontrado em estudo de Silva et al. ${ }^{17}$, no qual a resposta da FC também se apresentou menor quando os voluntários foram submetidos à aplicação de EPAP com pressão equivalente a $5 \mathrm{cmH}_{2} \mathrm{O}$. Sena et al. ${ }^{18}$, em seu estudo, afirmam que a aplicação de EPAP com $10 \mathrm{cmH}_{2} \mathrm{O}$ é bem tolerado em pacientes no pós-operatório de cirurgia cardíaca, sem aumento significativo na FC. Neste sentido, em função da resposta semelhante da FC entre os diferentes níveis pressóricos, nosso estudo mostra que a utilização de níveis pressóricos maiores pode ser bem tolerada, caso haja indicação para o seu emprego. Contudo, a realização de estudos específicos em diferentes condições clínicas e/ou cirúrgicas pode ser necessária. Por outro lado, Cardoso et al. ${ }^{19}$ realizaram estudo com pacientes portadores de DPOC e observaram que a aplicação de EPAP com $15 \mathrm{cmH}_{2} \mathrm{O}$ ajuda a reduzir o trabalho respiratório.

$\mathrm{Na}$ aplicação de EPAP no modo terapêutico, em relação ao nível "SHAM", os valores encontrados de $\triangle \mathrm{FC}$ foram menores $(\mathrm{p}<0,05)$ quando comparados aos níveis de EPAP de 15 e $20 \mathrm{cmH}_{2} \mathrm{O}$. No entanto, os valores de $\triangle \mathrm{FC}$ encontrados na modalidade SHAM mostraram-se muito próximos aos níveis de aplicação de EPAP na forma terapêutica de $10 \mathrm{cmH}_{2} \mathrm{O}$. Assim, provavelmente, pressões com valores iguais ou menores a $10 \mathrm{cmH}_{2} \mathrm{O}$ promovam alterações hemodinâmicas semelhantes ao uso isolado da máscara.

Apesar da diferença encontrada nas respostas da FC, identificou-se pequena variação dos valores de $\mathrm{PA}$ nas diferentes manobras (MV e EPAP). O emprego de metodologia indireta (método auscultatório) para aferição da PA pode ter contribuído para este resultado, tendo em vista que este apresenta limitações em relação à medida direta e contínua da PA, que é a metodologia padrão para a avaliação da resposta da PA durante a execução da $\mathrm{MV}^{20}$. A ausência dessa metodologia constitui uma limitação deste estudo. 
Outra limitação deste estudo decorre das diferenças metodológicas entre as manobras, relacionadas à duração e pressão utilizada. A técnica de EPAP possui duração média de 3 a 5 segundos e utiliza pressão máxima de $20 \mathrm{cmH}_{2} \mathrm{O}$, o que correspondente a cerca de $1 / 5$ da duração e menos da metade da resistência imposta durante a $\mathrm{MV}\left(54,3 \mathrm{cmH}_{2} \mathrm{O}\right)$. É possível que a aplicação de EPAP com maiores valores de pressão promova respostas hemodinâmicas mais próximas às encontradas durante a MV e, por isso, mereçam maior atenção por parte dos profissionais envolvidos na terapêutica.

Em função dos resultados observados, concluímos que a aplicação dos diferentes níveis pressóricos de EPAP, tanto na forma isolada quanto na forma terapêutica, não reproduz as respostas de FC observadas na execução da MV, em jovens saudáveis. No modo EPAP isolado, independentemente do nível pressórico, a resposta da FC é semelhante. Na modalidade EPAP terapêutico, apenas os valores de $\triangle \mathrm{FC}$ encontrados no nível $10 \mathrm{cmH}_{2} \mathrm{O}$ são semelhantes à aplicação SHAM, sem resistência pressórica. $\mathrm{Na}$ prática clínica, os dados referentes às respostas $\mathrm{da} \mathrm{FC}$ nos permitem inferir que a aplicação de EPAP é um procedimento terapêutico seguro nas condições estudadas, pois gera menor sobrecarga cardíaca.

\section{AGRADECIMENTOS}

Agradecemos ao $\mathrm{CNPq}$ pelo suporte financeiro (Processo no 503208/2008), à Prof ${ }^{a}$ Dra Ester da Silva (UNIMEP/UFSCar) pelo desenvolvimento conjunto do software de coleta dos dados de frequência cardíaca, e aos voluntários pela dedicação durante a realização do estudo.

\section{REFERÊNCIAS}

1. Publio AZ, Tuzi DA, Possoni HC, Rocha SM. Estudo da pressão positiva expiratória a sua eficácia na atelectasia: uma revisão literária. Fisioter Mov. 2004:17(4):43-50.

2. Scanlan CL, Myslinski, M.J. Terapia de higiene brônquica. In: Scanlan CL, Wilkins RL, Stoller JK. Fundamentos da terapia respiratória de Egan. 7a ed. São Paulo: Manole; 2000. p.817-43.
3. AARC Clinical Practice Guideline. Use of Positive Airway Pressure Adjuncts to Bronchial Hygiene Therapy. Respir Care. 1993;38(5):516-21.

4. Marães VRFS, Santos MDB, Catai AM, Moraes FR, Oliveira L, Gallo Jr L, et al. Modulação do sistema nervoso autonômico na resposta da freqüência cardíaca em repouso e à manobra de Valsalva com incremento da idade. Rev Bras Fisioter. 2004;8(2):97-103.

5. Looga R. The Valsalva Manoeuvre - cardiovascular effects and performance technique: a critical review. Respir Physiol Neurobiol. 2005;147(1):39-49.

6. Lárez MAM, Rangel NAJ, Oca, IM. Insuficiencia cardiaca: utilidad de la maniobra de Valsalva. Med Interna. 2004;20(1):38-47.

7. Liang, F; Liu, H. Simulation of hemodynamic responses of the Valsalva Maneuver: an integrative computational model of the cardiovascular system and the autonomic nervous system. J Physiol Sci. 2006;56(1):45-65.

8. Hanly PJ, George CF, Millar TW, Kryger MH. Heart rate response to breath-hold, Valsalva and Mueller maneuvers in obstructive sleep apnea. Chest. 1989;95(4):735-39.

9. Silva E, Catai AM, Trevelin LC, Guimarães JO, Silva Jr LP, Silva LMP, et al. Design of a computerized system to evaluate the cardiac function during dynamic exercise. Phys Med Biol. 1994;33(A):409.

10. Looga R. The bradycardic response to the Valsalva manoeuvre in normal man. Respir Physiol. 2001;124(3):205-15.

11. O'Brien IAD, O'Hare P, Corrall RJM. Heart rate variability in healthy subjects: effects of age and the derivation of normal ranges for tests of autonomic function. Br Heart J. 1986;55(4):348-54.

12. Gelber DA, Pfeifer M, Dawson B, Schumer M. Cardiovascular autonomic nervous system tests: determination of normative values and effect of confounding variables. J Auton Nerv Syst. 1997;62(1-2):40-44.

13. Treiber FA, Kamarck T, Schneiderman N, Sheffield D, Kapuku G, Taylor T. Cardiovascular reactivity and development of preclinical and clinical disease states. Psychosom Med. 2003;65(1):46-62.

14. Elghozi JL, Girard A, Fritsch P, Laude D, Petiprez JL. Tuba players reproduce a Valsalva maneuver while playing high notes. Clin Auton Res. 2008;18(2):96-104.

15. Naughton MT, Floras JS,Rahman MA, Jamal M, Bradley TD. Effect of continuous positive airway pressure on intrathoracic and left ventricular transmural pressure in patients with congestive heart failure. Circulation. 1995;91(6):1725-31.

16. Denault AY, Gasior TA, Gorcsan J, Mandarino WA, Deneault LG, Pinsky RM. Determinants of aortic pressure variation during positivepressure ventilation in man. Chest. 1999;116(1):176-86.

17. Silva FMF, Bagnall MES, Zardo TS, Bovi A, Carvalho EM, Lopes CR. Repercussões hemodinâmicas e ventilatórias em indivíduos sadios com diferentes níveis de EPAP. Fisioter Mov. 2009;22(3):419-26.

18. Sena ACBS, Ribeiro SP, Condessa RL, Vieira SRR. Expiratory positive airway pressure in post operative cardiac hemodynamics. Arq Bras Cardiol. 2010;95(5):594-99.

19. Cardoso DM, Paiva DN, Albuquerque IM, Jost RT, Paixão AV. Efeitos da pressão positiva expiratória nas vias aéreas sobre a atividade eletromiográfica da musculatura acessória da inspiração em portadores de DPOC. J Bras Pneumol. 2011;37(1):46-53.

20. Castro CLB, Nóbrega ACL, Araújo CGS. Testes autonômicos cardiovasculares. Uma revisão crítica - parte II. Arq Bras Cardiol. 1992;59(2):151-158. 